\title{
"Drug resistance pattern, related socio- demographic factors and preventive practices among MDR-TB patients: An experience from a tertiary care setting."
}

\author{
Dr Nirmalya Manna ${ }^{1}$, Kajaree Giri ${ }^{1}$, Dr Malay Mundle ${ }^{1}$ \\ ${ }^{I}$ Department of Community Medicine, Medical College, Kolkata, India
}

\begin{abstract}
The emergence of resistance to anti-tubercular drugs in general is a significant public health problem that threatens Tuberculosis care and control worldwide. An observational, cross-sectional, hospital based study was conducted among MDR-TB patients to know the drug resistance pattern and the preventive practices adopted by them. A pre-designed, semi-structured questionnaire was used for collection of data. $53.7 \%$ patients were found to be resistant to Rifampicin only, $46.3 \%$ to both Rifampicin and Isoniazid. All the patients were nonreactive to ICTC testing. 53.6\% of patients disposed off their sputum/ expectorations at a pot kept at bedside and later disposed at bathrooms. $29.2 \%$ spitted at roadsides and rest in their toilets. Health education regarding spread of disease, early detection of MDR-TB by strengthened laboratory support, effective therapy, implicating innovative control measures, and applying them specially among immigrants, would interrupt the ongoing transmission and control this emerging epidemic.
\end{abstract}

Key Words: MDR-TB, drug resistance, sputum disposal, Anti tubercular treatment

\section{Introduction}

Tuberculosis(TB), a disease caused by Mycobacterium tuberculosis, has affected mankind for over 5000 years, and still continues to be a leading cause of morbidity and mortality. ${ }^{1}$ The emergence of resistance to anti-tubercular drugs in general is a significant public health problem that threatens tuberculosis care and control worldwide. Multi-drug-resistant tuberculosis (MDR-TB) is defined as tuberculosis that is resistant to at least Isoniazid (INH) and Rifampicin (RMP), the two most powerful first-line treatment anti-TB drugs. MDR-TB results from infection with already drug-resistant bacilli or may develop during a patient's treatment, mostly due to administration of improper regimens in chemotherapy of drug-susceptible TB patients and failure to ensure patient compliance. Laboratory facilities for drug susceptibility testing are inadequate, thus diagnosis is often missed, and the actual number of global drug resistant tuberculosis cases remains unestimated. ${ }^{2}$ WHO estimates that $60 \%$ of the MDR-TB cases occurring among all TB cases notified worldwide in 2011 hail from Brazil, China, India, the Russian Federation and South Africa alone ("BRICS" countries). ${ }^{3}$ About $3.7 \%$ of new tuberculosis patients in the world have multidrug-resistant strains and $20 \%$ among those who are previously treated. ${ }^{3}$ About $4 \%$ of new TB cases have been reported with results for drug resistance testing, which is $6 \%$ among those previously treated. ${ }^{3}$ One in five of the estimated MDR-TB cases have been enrolled on treatment in the world and $48 \%$ of patients with MDR-TB enrolled on treatment in 2009 were successfully treated. ${ }^{3}$ Multidrug resistant TB requires treatment that needs two years or more using more toxic and expensive drugs, financially crippling the health care resources in tuberculosis endemic areas and complicating patient management. The advent of HIV/AIDS contributed to these substantially. ${ }^{1}$ Epidemiological studies for MDRTB is important to optimize drug therapy and prevent the dissemination of resistant strains in the community. With this background the present study was undertaken with the following objectives:

\section{Aims And Objectives}

1. To study the socio-demographic profile of the study population.

2. To assess the drug resistance pattern and the preventive practices adopted by the patients.

3. To identify the HIV status of the above mentioned patients.

\section{Methodology}

It was an observational, cross-sectional, hospital based study among MDR-TB patients admitted in K.S Roy (Kumud Sankar Roy) Tuberculosis Hospital, located at 1F, Raja Subhodh Chandra Mullick Road, Jadavpur, Kolkata -700032, West Bengal. The duration of study was six months. All MDR-TB positive patients admitted in the MDR-TB ward (NRS ward and Narayani ward) of the above-mentioned hospital during the study tenure constituted the study population. Inclusion criteria were Sputum positive tuberculosis patients with proven Isoniazid and Rifampicin resistance and no history of hepatic or renal complications. Unconscious patients and children below 10 years of age were excluded from the study. A pre-designed, semi-structured 
questionnaire was prepared including topics related to age, sex, education, housing environment (overcrowding, adequate ventilation), type of tuberculosis detected, whether received any anti-tubercular drugs before detection of multidrug resistance, any change in category during course of treatment, reason behind such change, mean duration of Anti Tubercular Treatment (ATT) taken before start of category IV, any side effects experienced by the patients during ATT, methods of sputum disposal, patterns of drug resistance, HIV status, clinical symptoms and their respiratory system examination findings. Then it was pre-tested in few patients not included in the study and modifications were made accordingly. The hospital authority was contacted and the purpose of the study was explained. After obtaining the permission from them, the investigator visited the MDR-TB wards as per pre-planned schedule for acquiring information from the patients. Adequate precautions were taken by the investigator to prevent acquiring disease. Mutually suitable days of the week were fixed when the patients felt fine and had no any prior commitments with their relatives or for investigations. The patients were explained about the purpose of the study, and assured of confidentiality. A verbal consent was obtained from them before personally questioning each one of them meeting the inclusion criteria of the study. This was followed by a session educating the patients on their disease, methods of safe sputum disposal, techniques to reduce transmission, and importance of proper diet and nutrition within their means. Questions and concerns of the patients, if any, were addressed. The responses of the patients were then compiled in an Excel sheet and analyzed by using Epi info and SPSS software package. Relevant statistical tests were applied to study the test of significance.

\section{Results}

41 patients are interviewed and examined from the NRS ward (29 male MDR-TB patients enrolled under Government therapy) and Narayani ward (12 female MDR-TB patients) of K.S.Roy About $46.3 \%$ of the study population were in their $3^{\text {rd }}$ decade $\left(20-29\right.$ years), followed by $11(26.8 \%)$ patients in their $4^{\text {th }}$ decade. Five patients were less than 19 years old. $41.5 \%$ have completed their primary education and $39 \%$ had no formal education. An equal number of patients that is $26.8 \%$ (11 each) had their BMI less than $15 \mathrm{~kg} / \mathrm{m}^{2}$ and between $16-18.5 \mathrm{~kg} / \mathrm{m}^{2}$ during treatment. $43.9 \%$ were skilled labourers (bus drivers, conductors, shopkeepers, grocery men, chef, plumbers, masons) or service men, $19.5 \%$ were unemployed and $9.8 \%$ were students and $12.2 \%$ were factory workers. Out of women, $72 \%$ were home makers and 2 are students. $75.6 \%$ were urban population with $48.7 \%$ having more than 5 family members, and $41.5 \%$ had $3-5$ family members. $31.7 \%$ belonged to socioeconomic class III and $24.4 \%$ in class IV. $24 \%$ were addicted to both tobacco and alcohol for more than 10 years. $36.6 \%$ have single child, $19.5 \%$ had 2 children each. $7.3 \%$ had 3 and more children each. Rest were unmarried. $78 \%$ of the patients did not have cross ventilation in their house. Clinical profile of patients and adverse effects of drugs were given in table 2 . About 15 patients reported other clinical symptoms like chest pain (pleuritic), breathlessness (grade II/III), myopathy, weight loss, vertigo at the onset of disease. Out of 22 patients who had hemoptysis, $12(54.5 \%)$ had their first episode after initiating or completing category I drugs. One patient had tubercular meningitis during the course of treatment. 5 patients had cervical lymphadenopathy more than $1 \mathrm{~cm}$ in each case. They were at level Ib, II, discrete, soft, not fixed to underlying structures or skin. One female patient presented with hematuria, dysuria and following cystoscopy was diagnosed as a case of urinary bladder tuberculosis. She later developed right sided pleuritic chest pain, breathlessness. Four patients gave history of contact with tuberculosis patients, at least 2 years before their onset of symptoms. The sources had been adequately treated by category I drugs and were reported well till date, except one (aged 51years) who died due to tuberculosis. Regarding adverse effects of drugs, 15 patients reported of other adverse effects like headache, vertigo, syncope, joint pain, tingling and numbness of extremities, diarrhoea on receiving Category I/II drugs. 12 patients (29.3\%) reported no side effects and one complained of deafness post streptomycin injection. $73.2 \%$ patients were initially started on category I regimen, then shifted to category II regimen and then received MDR drugs, while 26.8\% received MDR drugs after Category I therapy only. Reasons for suspecting MDR-TB were given on table 3. 46.3\% received anti tubercular drugs (category I/ II/ both) for 1-2 years before starting MDR drugs and $26.8 \%$ had them for 6 months to 1 year only. $63.4 \%$ of them reported absolute compliance whereas $24.4 \%$ confessed forgetting medications at least once in a month. $61 \%$ received drugs from government centres only and $31 \%$ from both government and private hospitals. $65.9 \%$ ( 27) were tested drug resistance by LPA method, $24.4 \%$ (10) by CBNAAT method and rest by MGIT-960 method. About $53.7 \%$ (22) patients were found to be resistant to Rifampicin only, $46.3 \%$ (19) to both Rifampicin and Isoniazid. All the patients were nonreactive to ICTC testing. 53.6\% of patients disposed off their sputum/ expectorations at a pot kept at bedside, (some with dettol in it) and later disposed at bathrooms, 29.2 $\%$ spitted at roadsides and rest in their toilets. $68 \%$ of the patients received drug doses in the $26-45 \mathrm{~kg}$ weight band. On respiratory system examination, respiratory distress was complained by 12 patients, 2 had rhonchi and crepitations each, COPD was diagnosed among 8 patients. Among the 12 female patients, 7 complained of amenorrhoea or oligomenorrhoea following drug intake. 9 of them received previous hospital admissions due to breathlessness and 2 required pleural tapping. All of them had no addictions. $66 \%$ of them were resistant to both 
Isoniazid and Rifampicin compared to $37 \%$ of males. Five were breast feeding their babies when they were diagnosed tuberculosis and all of them continued breast feeding, with only one woman reported of using cloth on her mouth during feeding. Children of four of these women underwent Mantoux test, chest skiagram and received Isoniazid tablets from government centres.

\section{Discussion}

The prevalence of Multi drug resistance tuberculosis is on a rise since decades globally. The 3-month population-based incidence rate of MDR TB in New York City was 52.4 times (95\% confidence interval [CI], 35.5 to 78.3$)$ that of the rest of the nation ( 9.559 vs 0.182 cases per million) ${ }^{4}$. There is an extremely high burden of Multidrug resistant Tuberculosis among the BRICS countries which may be attributed to its demographic and socio-economic profile like poverty, lack of knowledge attitude and practice, overcrowding, malnutrition, care during illness and lack of social security. Adequate information on prevalence of MDR-TB, epidemiological factors and their interactions are essential prerequisites ${ }^{5}$ to redirect health resources in formulating a National Treatment policy which would control the transmission pattern of MDR-TB as well as ensure better patient management and its prevention. As no single factor is fully attributable for emergence of MDR-TB, and study regarding its epidemiology is scarce especially in eastern India, we have tried to explore different socio-demographic factors. In the present study majority of the patients $(46.3 \%)$ were in their third decade. A study in west Australia by Francis et al ${ }^{6}$ showed the median age of the MDR-TB patients were 26 years. However, in separate studies Ulmasova et al ${ }^{7}$ and Ndung'u et al ${ }^{8}$ showed that MDR-TB was significantly associated with age less than 45 years in Uzbekistan and Nairobi (88.7\%) respectively. Malnutrition since childhood leading to weakened immune response, made the younger population more susceptible to MDR-TB in developing countries. In the present study $39 \%$ had no formal education, $56.1 \%$ were employed and $19.5 \%$ were unemployed (excluding students). $78 \%$ had their income less than Rs 5433 monthly and $78 \%$ were suspected to have overcrowding at their homes. $24 \%$ was addicted to tobacco and alcohol for more than 10 years. $29.3 \%$ of open TB cases disposed off their sputum at roadsides. A study in Rawalpindi, Pakistan by Khurram et al ${ }^{9}$ showed similar results where among total, $60 \%$ patients were illiterate, $60 \%$ employed, $60 \%$ had income less than Rs 5000 (42 Euro per month), $73.3 \%$ lived in an overcrowded residence and $60 \%$ were smokers. Ndung'u et $\mathrm{al}^{8}$ showed $66.5 \%$ of the MDR-TB study population in Nairobi were unemployed and $79.4 \%$ earned less than 100 USD per month. MDR-TB estimates for Uzbekistan were among the highest reported in former Soviet Union countries which were attributed to factors like imprisonment, unemployment and previous treatment with TB as shown by Ulmasova et $\mathrm{al}^{7}$. Primarily resistant TB strains, malnutrition, poverty, overcrowding, poor hygiene, decreased health care seeking attitude formed a vicious cycle of agent-host- environment. Illiteracy contributed to poor awareness of disease transmission, proper method of sputum disposal and unemployment or poor income decreases health care seeking behaviour, proper care during illness. Addiction leads to wastage of money as well as adds to malnutrition and overcrowding facilitates ongoing transmission of MDR-TB strains. Four patients had history of contact with TB. Obtaining a detailed contact history is essential as a delay in starting appropriate MDR treatment has potentially serious consequences as shown by Schaaf et al ${ }^{10}$ in a study among MDR-TB children regarding diagnostic delay and outcome. Regarding adverse effects of ATD , gastrointestinal symptoms was reported by majority and headache vertigo were reported only by few, whereas, a study in Iran by Baghaei et al ${ }^{11}$, showed major side effects were neurological (depression, convulsion, psychotic, suicide) increasing the risk of death. 14.5 had auditory toxicity. This contrast in results may be accounted due to decreased number of study population, or due to the fact that, in India, mental health was not given enough significance till date and depression among patients often remained undiagnosed as well as untreated. In the present study, all the patients were previously treated with category I (26.8\%) or both I and II (73.2\%) regimen before being diagnosed MDR. 46.3\% received ATD for 1-2 years before starting on MDR therapy. Majority (34.1\%) were retreatment sputum positive cases at diagnosis of MDR. BMI of $26.8 \%$ of patients were less than $15 \mathrm{~kg} / \mathrm{m}^{2} .24 .4 \%$ reported noncompliance at least once a month. A Study in South India by Santharam et al ${ }^{12}$ showed primary and acquired resistances for both INH and RMP were $1.25 \%$ and $19 \%$ respectively, supporting a similar study in North India by Maurya et al ${ }^{13}$, who showed MDR-TB prevalence more in previously treated cases $(43.3 \%)$ than new cases $(29.1 \%)$. Al-hajoj et al ${ }^{14}$ found multi drug resistance in $1.8 \%$ and $15.9 \%$ of new and pre-treated cases of TB respectively in a study in Saudi Arabia and that presence of pulmonary TB, active TB were strongest independent contributors. Similar results were reported from Rawalpindi, Pakistan by Khurram et al ${ }^{9}$, and from Bangladesh by Banu et al ${ }^{15}$ who showed MDR significantly increase in those previously treated or defaulters. In Ethiopia, Abate $\mathrm{G}^{16}$ Showed MDR prevalence were more in retreatment cases $(12 \%)$ than new (1.2\%). In A study shown by Liang et al ${ }^{17}$ in China, inappropriate treatment, longer delays to effective therapy were the most influential factors behind emergence of drug resistance. Retreatment patients were 5.48 times and those with previous INH and RMP therapy for more than 180 days were 4.82 times more likely to develop MDR-TB. Similar result was given by Francis et al ${ }^{6}$ in West Australia. Drug resistance was checked by a rapid manual nucleic acid amplification test(NAAT)- LPA (Line 
probe assay) for $1^{\text {st }}$ and $2^{\text {nd }}$ line TB drugs. A real time PCR test - CBNAAT ( Cartridge based nucleic acid amplification test) and MGIT- 960 ( Mycobacterium growth indicator tube ) tests were also used. $53.7 \%$ patient in our study was resistant to Rifampicin only and $46.3 \%$ had both Isoniazid and Rifampicin resistance. In a study in north-west Ethiopia , by Tesseme et al ${ }^{18}, 13.8 \%$ were resistant to $\mathrm{INH}$, followed by $10 \%$ to Ethambutol, 5.8\% to Rifampicin and all were susceptible to all second line drugs. In Brazil, Lemos et al ${ }^{19}$ reported that with use of five drugs: Terizidone, Levofloxacin, Pyrazinamide, Ethambutol and an aminoglycoside (Streptomycin or Amikacin) for MDR-TB and Pulmonary resection as coadjuvant treatment an average cure rate of $69 \%$ has been achieved. In Nairobi, high resistance to INH $(30.2 \%)$ followed by streptomycin $(11.6 \%)$ has been observed ${ }^{8}$. In Pakistan, DST showed high resistance to Ethambutol, Pyrazinamide, Streptomycin in more than $60 \%{ }^{9}$. In Ethiopia uniform susceptibility to Ethambutol has been noted $^{16}$. This difference in patterns of drug resistance globally can be attributed to the variance in genomic structures of MDR-TB strains such as resistance to INH confers to gene inh A, katG, Rifampicin by rpoB gene, Pyrazinamide by pncA and so on .

\section{Conclusion}

This study strongly highlighted the need of a strengthened continuous surveillance system that monitors the trends of drug resistance over time and places. Drug sensitivity testing is recommended at least for previously treated cases, defaulters, patients who remain smear-positive at the end of the second month of treatment and patients in close contact with MDR-TB cases. This study has few limitations regarding time constraints, lesser number of study populations, but also has some positive aspects. Various socio-demographic factors like younger age, illiteracy, unemployment, poverty, overcrowding, lack of cross ventilation at home, addiction, decreased BMI, improper techniques of sputum disposal thus favouring disease transmission, were identified. Additionally, it was shown that drug resistance is more in defaulters and previously treated patients. Health education regarding spread of disease, early detection of MDR-TB by strengthened laboratory support, effective therapy, implicating innovative control measures, and applying them specially among immigrants, would interrupt the ongoing transmission and control this emerging epidemic. Issues such as poverty, malnutrition should be addressed, control of infections and improved hygiene and strong commitment and collaboration among health care providers should become a priority. Efforts to improve mental health need scaling up. Future longitudinal studies with a large sample may be conclusive of identifying the predictors of the disease that will contribute in other meta analytical studies or formulating national treatment policies.

\section{Acknowledgements}

We are thankful to the administration of K.S.Roy Hospital and the patients for their cooperation and support.

VII. Tables and Charts

Table 1: Socio-demographic profile of the study population

\begin{tabular}{|l|c|l|}
\hline \multicolumn{1}{|c|}{ Table 1: Socio-demographic profile of the study population } & Percentage \\
Variables & Frequency & \\
\hline Age in years & & 12.2 \\
$\leq 19$ & 5 & 46.4 \\
$20-29$ & 19 & 26.8 \\
$30-39$ & 11 & 14.6 \\
$\geq 40$ & 6 & 39.0 \\
\hline Formal Education & 16 & 41.4 \\
Nil & 17 & 9.8 \\
Completed primary & 4 & 9.8 \\
Completed secondary & 4 & \\
Higher secondary and above & & 9.8 \\
\hline Number of family members & 4 & 41.5 \\
<3 & 17 & 48.7 \\
3-5 & 20 & \\
$>5$ & & 22.0 \\
\hline Social Class (Prasad scale) & 9 & 22.0 \\
I (Rs5433 and above) & 9 & 31.7 \\
II (Rs 2716- 5432) & 13 & 24.3 \\
III (Rs 1630 - 2715) & 10 & \\
IV (Rs 815 -1629) & & \\
\hline
\end{tabular}


Table 2: Clinical profile of the study population

$$
(\mathrm{n}=41)^{*}
$$

\begin{tabular}{|c|c|c|}
\hline Variables & Frequency & Percentage \\
\hline $\begin{array}{l}\text { Clinical symptoms(during diagnosis) } \\
\text { Fever } \\
\text { Dry cough } \\
\text { Expectoration } \\
\text { Hemoptysis (blood spitting without cough) } \\
\text { Others }\end{array}$ & $\begin{array}{l}30 \\
22 \\
11 \\
22 \\
15\end{array}$ & $\begin{array}{l}73.1 \\
53.6 \\
26.8 \\
53.6 \\
36.5\end{array}$ \\
\hline
\end{tabular}

\section{*Multiple responses}

Table 3: Reasons for MDR -TB suspect

$(\mathrm{n}=41)$

\begin{tabular}{|l|l|l|}
\hline Reasons & Frequency & Percentage \\
\hline Category I failure & 12 & 29.3 \\
Category II sputum positive at $>4$ months & 12 & 29.3 \\
Retreatment sputum positive at diagnosis & 14 & 34.1 \\
Any follow-up sputum positive in new/retreatment case & 3 & 7.3 \\
\hline
\end{tabular}

Table 4: Preventive practices adopted by the patients

\begin{tabular}{|l|l|l|}
\hline Method of sputum disposal & Frequency & Percentage \\
\hline Roads & 12 & 29.2 \\
Pots at bedside & 22 & 53.6 \\
Bathroom & 6 & 14.6 \\
Packets & 1 & 2.6 \\
\hline
\end{tabular}

Table 5: Drug resistance pattern among study population

$(\mathrm{n}=41)$

\begin{tabular}{|l|l|l|}
\hline Variables & Frequency & \\
\hline Drug resistance pattern & & \\
& 22 & \\
Resistance to Rifampicin only & 19 & 53.6 \\
Resistance to both Rifampicin and Isoniazid & & 46.4 \\
\hline MDR culture methods & 27 & \\
LPA & 10 & 65.8 \\
CBNAAT & 4 & 24.4 \\
MGIT 960 & 9.8 \\
\hline
\end{tabular}

\section{References}

[1]. Sarala Menon, Sujata Dharmshale, Chhaya Chande, Aruna Gohil, Sunil Lilani, Salim Mohammad, et al. Drug resistance profiles of Mycobacterium tuberculosis isolates to first line anti-tuberculous drugs: A five years study. Lung India 2012;29(3): 227-31.

[2]. Zumla A, Abubakar I, Raviglione M, Hoelscher M, Ditiu L, McHugh TD, et al. Drug -resistant-tuberculosis- current dilemmas, unasnswered questions, challenges and priority needs. J Infect dis. 2012;205(suppl 2):S228 -40. Epub 2012 Apr 3.

[3]. Open database : WHO's certified [database on the internet]. Multidrug-resistant TB (MDR-TB): 2012 Update.

[4]. Bloch AB et al. Nationwide survey of drug-resistant tuberculosis in the United States. JAMA. 1994 Mar 2;271(9):665-71.

[5]. Bhatt G, Vyas S, Trivedil K. An epidemiological study of multi drug resistant tuberculosis cases registered under Revised National Tuberculosis Control Programme of Ahmedabad City. Indian J Tuberc. 2012 Jan; 59(1):18-27.

[6]. Francis $\mathrm{JR}^{1}$, Blyth $\mathrm{CC}^{2}$, Colby $\mathrm{S}^{2}$, Fagan $\mathrm{JM}^{2}$, Waring $\mathrm{J}^{2}$. Multidrug-resistant tuberculosis in Western Australia, 1998-2012. Med J Aust. 2014 Apr 7;200(6):328-32.

[7]. Ulmasova DJ et al. Multidrug-resistant tuberculosis in Uzbekistan: results of a nationwide survey, 2010 to $2011 . \quad$ Euro Surveill. 2013 Oct 17;18(42). pii: 20609.

[8]. Ndung'u PW ${ }^{1}$, Kariuki S, Ng'ang'a Z, Revathi G. Resistance patterns of Mycobacterium tuberculosis isolates from pulmonary tuberculosis patients in Nairobi. J Infect Dev Ctries. 2012 Jan 12;6(1):33-9.

[9]. Khurram $\mathrm{M}^{1}$, Khaar HT, Fahim M. Multidrug-resistant tuberculosis in Rawalpindi, Pakistan. J Infect Dev Ctries. 2012 Jan 12;6(1):29-32.

[10]. Schaaf $\mathrm{HS}^{1}$, Shean K, Donald PR. Culture confirmed multidrug resistant tuberculosis: diagnostic delay, clinical features, and outcome. Arch Dis Child. 2003 Dec;88(12):1106-11.

[11]. Baghaei $\mathrm{P}$ et al. Adverse effects of multidrug-resistant tuberculosis treatment with a standardized regimen: a report from Iran. Am J Ther. 2011 Mar-Apr;18(2):e29-34. 
[12]. Santharam P, Appalaraju B. Incidence of multidrug resistance in Mycobacterium tuberculosis in a tertiary care hospital in South India--a prospective study. J Commun Dis. 2012 Sep;44(3):151-5.

[13]. Maurya AK et al. Changing patterns and trends of multidrug-resistant tuberculosis at referral centre in Northern India: a 4-year experience. Indian J Med Microbiol. 2013 Jan-Mar;31(1):40-6.

[14]. Al-Hajoj S et al. Epidemiology of antituberculosis drug resistance in Saudi Arabia: findings of the first national survey. Antimicrob Agents Chemother. 2013 May;57(5):2161-6. Epub 2013 Mar

[15]. Banu $\mathrm{S}$ et al. Multidrug-resistant tuberculosis in admitted patients at a tertiary referral hospital of Bangladesh. PLoS One. 2012;7(7):e40545. Epub 2012 Jul 11.

[16]. Abate G. Drug-resistant tuberculosis in Ethiopia: problem scenarios and recommendation. Ethiop Med J. 2002 Jan;40(1):79-86.

[17]. Liang L et al. Factors contributing to the high prevalence of multidrug-resistant tuberculosis: a study from China. Thorax. 2012 Jul;67(7):632-8. Epub 2012 Mar 8.

[18]. Tessema B ${ }^{1}$, Beer J, Emmrich F, Sack U, Rodloff AC. First- and second-line anti-tuberculosis drug resistance in Northwest Ethiopia. Int J Tuberc Lung Dis. 2012 Jun;16(6):805-11. Epub 2012 Feb 29.

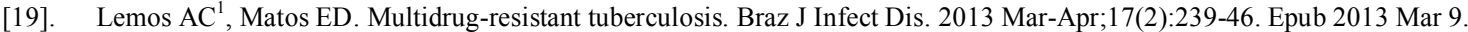

